\title{
Assessing effectiveness of a novel mid-upper arm circumference Z-score tape in a community setting in Guatemala
}

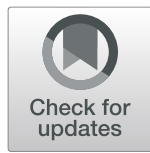

Mikaela A. Miller ${ }^{1 *}$ (D) Kristen Mallory ${ }^{1}$, Manolo Escobedo ${ }^{2}$, Ana Cecilia $\operatorname{Tarot}^{2}$ and Susan Abdel-Rahman ${ }^{3}$

\begin{abstract}
Background: Mid-Upper Arm Circumference (MUAC) is an independent anthropometric measurement used to identify malnutrition in children. While much research has been dedicated to applying fixed estimates of MUAC to identify cases of malnutrition in children under 5 years of age, far less has been done with age-specific MUAC $Z$-score values across the continuum of age from birth through adolescence.

Methods: The present study examined the effectiveness of a novel MUAC Z-score tape, in the hands of community health volunteers, to identify children over the age of 5 who would benefit from nutritional rehabilitation. In January of 2019, 112 community health volunteers working within Children International in Guatemala were trained to use the MUAC Z-score tape and asked to collect measurements on children or youth in their communities.

Results: Of the 818 MUAC Z-score tape measurements obtained by volunteers, 88.26\% (722/818) were concordant with nutritional risk status as predicted by BMI Z-score, and 90.95\% (744/818) were concordant with MUAC Z-score tape measurements made by field medical staff. MUAC Z-scores identified $87.10 \%$ (27/31) of the severely or moderately undernourished children as determined by the BMI Z-score who would be candidates for the nutrition rehabilitation program (Z-score $\leq-2$ ) along with an additional six children that would not have been classified as such with BMI Z-score. A qualitative survey distributed to the volunteers showed moderate rates of understanding of nutritional risk using the tape, and $62.50 \%$ reported the tape was easy to use.
\end{abstract}

Conclusions: These quantitative and qualitative findings suggest that with more in-depth training and education the MUAC Z-score tape is a viable, low-cost, low-burden alternative for community-level nutritional status assessment among the population served by Children International in Guatemala.

Keywords: Acute malnutrition, Mid-upper arm circumference, Community screening of malnutrition

\section{Background}

Accurate and timely identification of children suffering from, or at risk of, severe acute malnutrition (SAM) is essential to direct them to appropriate care. The deleterious and potentially irreversible effects of undernutrition on growth and cognitive development have been well documented in children under the age of five, and an estimated $45 \%$ of mortality in this age range is attributable to undernutrition [1-4]. Although most intervention work focuses on the critical window from conception to 24 months, an expanding body of evidence suggests that

\footnotetext{
* Correspondence: mmiller@children.org

${ }^{1}$ Children International, Kansas City, USA

Full list of author information is available at the end of the article
}

growth catch-up can occur during middle and late childhood and adolescence [5]. This period of life is marked by significant physical, neurological, and social development. Executive neurological function and frontal lobe development is thought to occur in late childhood and adolescence, and adequate nutrient intake is highly associated with cognitive performance and maintenance, as well as educational achievement [6-8]. Physiologically, underweight school-aged children are more likely to suffer from delayed onset of puberty, reduced bone density, deficient muscular development, and poorer overall health which may impact their ability to work later in life [9]. The effects of malnutrition persisting into adolescence and the reproductive years can extend to subsequent generations,

(c) The Author(s). 2019 Open Access This article is distributed under the terms of the Creative Commons Attribution 4.0 International License (http://creativecommons.org/licenses/by/4.0/), which permits unrestricted use, distribution, and 
adversely affecting fetal development and the epigenome of their children [10]. Thus, undernutrition in school-aged children and adolescents exerts considerable strain on the health, education, and economic systems in low and middle income countries [11].

Undernutrition is most prevalent in low-resource settings where unskilled staff, inadequate tools, and insufficient time hinder nutritional screening program success. Detection methods should, therefore, be simple for delivery at the community level [12]. Various anthropometric measurements have been utilized to identify high risk cases, of which Mid-Upper Arm Circumference (MUAC), Weight-for-Height (WH) Z-scores in children under 2, and Body Mass Index (BMI) Z-score for ages 2-19 are the most widely used and debated [13, 14]. MUAC, as measured in $\mathrm{mm}$, is an easier method to deploy in a community setting. WH/BMI Z-scores are utilized more often in health care settings where the tools required for obtaining weight and height are accessible. The cutoffs using WH/BMI Z-scores are well-established: SAM is identified by a Z-score $<-3$, and moderate acute malnutrition (MAM) is identified as a Z-score between -2 and -3 [15]. Cutoffs for SAM and MAM using traditional MUAC tapes are much less consistent. A systematic review in 2013 concluded that MUAC could be adequately used as a stand-alone criterion for hospital admission and discharge due to SAM, but the MUAC cutoffs ranged from 110 to $130 \mathrm{~mm}$ for children aged 6 to 59 months [16]. Furthermore, independent studies offer contradictory evidence as to which measurement is more predictive of mortality due to malnutrition. Chiabi et al. concluded that MUAC was more predictive of mortality in children with SAM than WH Z-score [17], whereas Grellety and Golden recently determined that current MUAC methods based on mm cutoffs for children under the age of 5 inadequately identified individuals with the highest risk of mortality based on WH [18]. Briend et al. found that MUAC was preferable to $\mathrm{WH}$ but did not add predictive value [19]. The disparities may be attributable in part to the various cutoffs used by each group. Other studies have attempted to determine more appropriate cutoffs in various populations using an endpoint other than mortality, but these were based solely on the MUAC expressed in $\mathrm{mm}$, and/or made for use in children ages 0-5 [20-29]. Accordingly, trade-off between ease of implementation in resource-limited settings, unacceptable rates of detrimental false negatives, and the burden of false positives needs to be addressed so that better protocol can be established and scaled.

To address limitations of traditional MUAC, several groups independently developed reference growth curves for age-specific MUAC Z-scores using the Health Examination Survey (HES) and the National Health and
Nutrition Examination Survey (NHANES) [30-32]. Application to an East African population offered evidence that MUAC Z-score growth curves were at least as effective as BMI Z-score in 5-19 year-olds with or without comorbid HIV infection. Data from U.S. cohorts extended the MUAC Z-score to include children aged 2 months to 20 years, and provided the first indication that MUAC Z-score and BMI Z-score thresholds may require refinement [30, 33]. To facilitate screening, the Kansas City group also constructed MUAC Z-score tapes that use easily interpretable colors to indicate nutritional risk status. The MUAC Z-score tapes have been evaluated in practice by registered dietitians on a group of over 10 , 000 patients seen at their institution between October 2015 and October 2017 [33].

The MUAC Z-score tape has yet to be evaluated in the hands of health volunteers in a resource-limited community setting. Increasing accessibility of the MUAC tapes for community health workers and volunteers is an important step to increase program coverage, both in detecting SAM and treating uncomplicated SAM in a community setting [34]. Notably, there is some evidence to suggest that mothers are able to more accurately take and read traditional MUAC for their children than community health workers, which indicates household community monitoring may be as or more effective than community health worker screening [35]. The present study examined the effectiveness of the MUAC Z-score tape described above [32, 33] in the hands of non-medical volunteers to identify children over the age of 5 who would benefit from nutritional rehabilitation $(\mathrm{Z}-$ score $<-2)$.

\section{Methods \\ Device}

Two versions of the MUAC Z-score tape were available for use, an infant tape spanning 2-59 months and one for children spanning 5-18 years. For this study we used the children's tape with discrete age groups of $5,5 \frac{1}{2}, 6$, $6 \frac{1}{2}, 7,7 \frac{1}{2}, 8,8 \frac{1}{2}, 9,9 \frac{1}{2}, 10,10 \frac{1}{2}, 11,12,13,14,15,16$, 17, and 18 years (see Additional file 1). The device was constructed using flexible, tear-resistant paper and printed by Hallmark Cards, Inc. (Kansas City, MO). Down the center of the device is a traditional measuring scale depicting centimeters and millimeters. Above and below this scale are a series of color-coded bands demarcating the Z-score range into which the child's MUAC falls for each age group. Markings appear on both sides and genders were pooled to mitigate the need for multiple versions of the device. After production, the tapes were checked for dimensional accuracy using a National Institute of Standards and Technology (NIST) certified ruler in compliance with ISO 9000 standards. 


\section{Study design}

Data were collected at the Tierra Nueva community center in the Guatemala agency of Children International (CI). CI is a mission-driven organization based in Kansas City, MO, USA that works in ten countries to end poverty through local partnerships, child and youth programming, and community involvement in the areas of health, education, empowerment, and employment. Tierra Nueva is one of seven community centers in the CI Guatemala agency and is located in Guatemala City. The population served by this community center is primarily urban and between 5 and 19 years of age.

$\mathrm{CI}$ health volunteers, primarily comprised of mothers and caregivers, were trained to use the MUAC Z-score tape at the Tierra Nueva community center. Training consisted of a 3-h session on the morning of January 11, 2019. Each volunteer received a MUAC Z-score tape and an instructional document that described how to use and interpret the tape. The Field Medical Officer presented general theory behind the tape and answered volunteers' questions. The volunteers then practiced using the tape on each other before breaking into small groups to practice with children who were present at the community center. During this practical training portion those volunteers who were adept with the tape served as coaches for the others in the sector (approximately 15 volunteers per sector). Volunteers were trained on how to complete the tracking sheet, data collection protocols, and the plan for the experiment. Each volunteer was asked to complete measurements in children over the age of 5 years in their communities and instructed to record the data per the protocol over the next two weeks.

MUAC Z-score tape color and measurements in $\mathrm{mm}$ were collected on paper by the health volunteers, and the data entered into a spreadsheet template by the Field Health Coordinator. The same children were then invited to the community center to obtain current height, weight, and MUAC Z-score tape measurements within 15 days, to be completed by the field medical staff. All health volunteers were invited to complete a survey about their experiences using the MUAC Z-score tape (see Table 2).

\section{Statistical analysis}

Standard descriptive statistics were used to summarize demographic and anthropometric characteristics. Continuous variables are reported with the median and interquartile range. Categorical variables are reported as percentage and counts represented by each level. BMI Z-scores were calculated for participants using the World Health Organization's (WHO) Child Growth Standards macro in R [36]. BMI Z-scores were categorized into 7 risk categories based on standard deviation intervals derived from the WHO 2007 growth standards [15] (Table 1).
Table 1 Z-score risk category ranges for the study

\begin{tabular}{ll}
\hline Risk category & $Z$-score range \\
\hline Severely malnourished & $Z<-3$ \\
Moderately malnourished & $-3 \leq Z<-2$ \\
At risk of underweight & $-2 \leq Z<-1$ \\
Normal & $-1 \leq Z<1$ \\
At risk of overweight & $1 \leq Z<2$ \\
Overweight & $2 \leq Z<3$ \\
Obese & $Z \geq 3$ \\
\hline
\end{tabular}

Agreement between pairs of anthropometric measurements concerning nutritional status (as predicted by BMI Z-score, MUAC Z-score range color measured by field medical staff, and MUAC Z-score range color measured by health volunteers) was assessed using Cohen's Weighted Kappa for ordinal responses (quadratic weights). Adjusted bootstrap confidence intervals for Cohen's Weighted Kappa were calculated using the BCa bootstrap method [37] with a P-value for Cohen's Weighted Kappa of 0.05 or less indicating significant statistical agreement. McNemar's Test of concordance (continuity corrected) was used to assess agreement between volunteer and BMI-predicted nutritional status in identifying nutrition rehabilitation candidates. Rejection of the null hypothesis for McNemar's Test would provide sufficient evidence to conclude disagreement.

Characteristics of the child or health volunteer were examined using a generalized linear mixed model (link $=$ logit) to determine if there was an association with misclassification and any of these factors. A random intercept was included for each health volunteer to account for intra-rater covariance. Polynomial terms for BMI Z-score were also examined up to degree 3. The normal approximation $(\operatorname{Pr}|\mathrm{Z}|)$ was used to evaluate the significance of individual factors. An alpha-level of 0.05 was used to determine statistical significance for all tests. The statistical package $R$ was used to conduct the analysis (version 3.5.2).

\section{Results}

\section{Tierra Nueva quantitative results}

In total, 112 volunteers were trained and 56 obtained MUAC Z-score tape measurements on 889 children and youth, of which 818 were able to complete follow-up height and weight measurements during the study duration. The remaining volunteers who did not contribute data to the study live in sectors with mostly older youth who would not have been considered candidates for the nutritional rehabilitation program. The median number of measurements completed by each community volunteer was 13.00 (IQR $=7.00-20.00$ ). Child age ranged from 6 to 13 years (median $=10.75$ years, IQR $=9.25-12.00$ ). There 
were slightly more females $(424 / 818,51.83 \%)$ than males (394/818, 48.17\%) that were measured with the MUAC Zscore tapes. Evidence of stunting was not as prevalent in the Tierra Nueva population (147/818, 17.97\%), though Guatemala has one of the highest rates of stunting in the Western Hemisphere [38].

The highest level of concordance (96.33\%, 788/818) was observed between the BMI Z-score predicted risk category and the MUAC Z-score category as measured by the field medical staff. The result was statistically significant with near-perfect agreement $(K=0.95 ; Z=27.18$, 95\% CI $(0.92,0.97), P \leq 0.0001)$ (Fig. 1). A concordance rate of $91.00 \%(744 / 818)$ was observed between MUAC $\mathrm{Z}$-score tape measurements made by volunteers and those made by the field medical staff (Fig. 2), suggesting an exceptionally high level of statistically significant agreement $(\mathrm{K}=0.88 ; \mathrm{Z}=25.26,95 \%$ CI $(0.85,0.91)$, $P \leq 0.0001$ ). We observed a concordance rate of $88.26 \%(722 / 818)$ with nutritional status predicted by MUAC Z-score as measured by the health volunteer versus BMI Z-score (Fig. 3). The results indicate a high level of agreement between nutritional status predicted by these two anthropometric measurements, albeit the lowest of the pairwise comparisons $(\mathrm{K}=$ $0.84 ; \mathrm{Z}=24.13,95 \%$ CI $(0.80,0.87), P \leq 0.0001)$.

Across all nutritional categories, we did not conclude significant disagreement between the nutritional status assigned by BMI Z-score and that assigned by MUAC Z-score tape $\left(\chi^{2}(1)=0.10, P=0.75\right)$ with a concordance rate of $98.78 \%(808 / 818)$. In terms of correctly identifying candidates for the nutrition rehabilitation program, we concentrated on the agreement between BMI Z-score and MUAC Z-score for SAM and MAM as a combined category (Table 2). BMI Z-score assigned 31 children to MAM/SAM whereas MUAC Z-score assigned 33 children to MAM/SAM, with 27 children categorized by both measures. The individuals missed by the MUAC Zscore tape were all labeled as "at risk" of undernutrition according to predicted BMI Z-score, and two of the 4 were measured by the same volunteer. The MUAC Z-score tape identified an additional 6 individuals as potential candidates for the nutrition rehabilitation program that BMI z-score did not

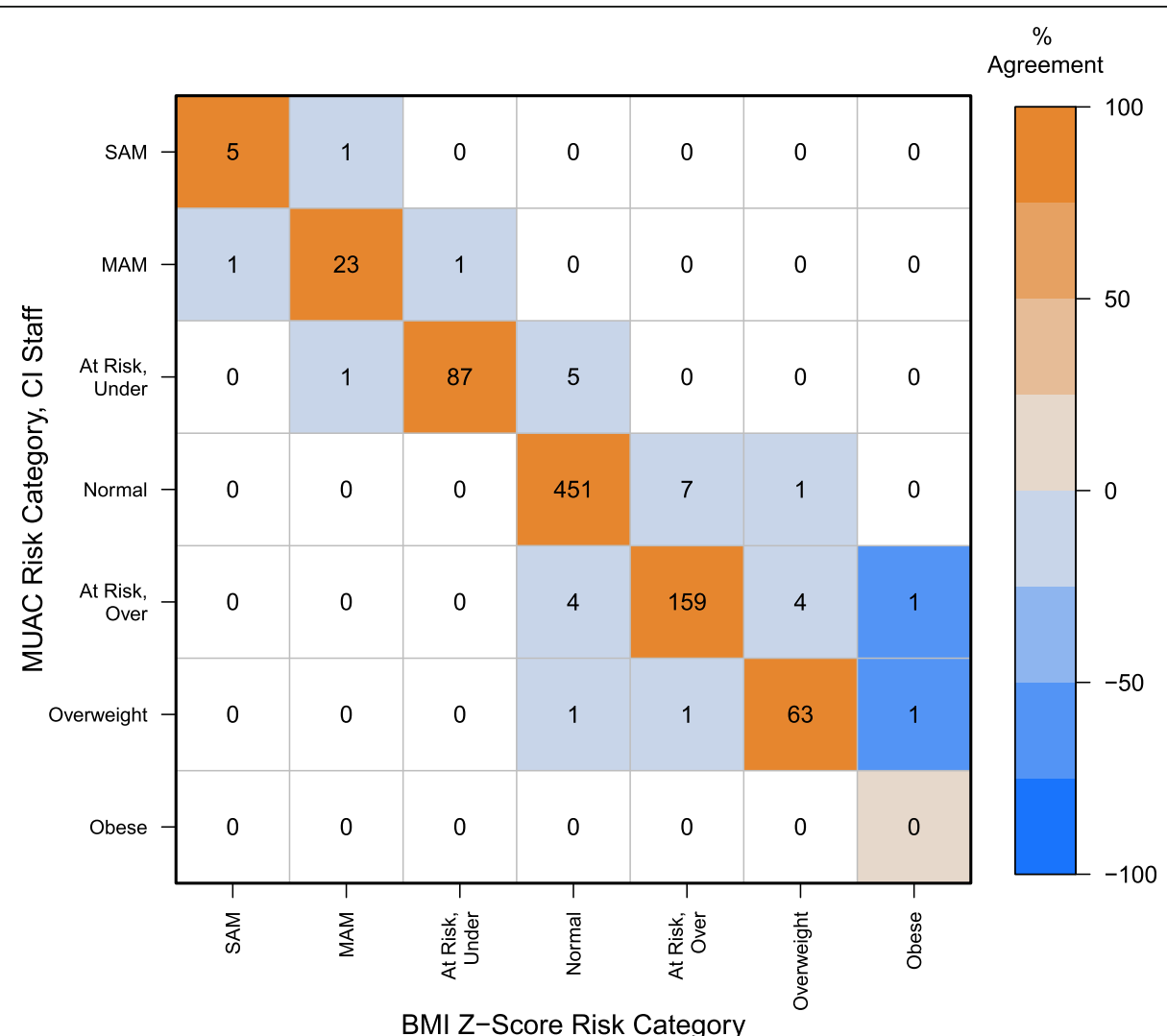

Fig. 1 Predicted BMI Z-score and MUAC Z-score risk categories as measured by Cl staff. The confusion matrix displays the counts in the cells, and color indicates agreement. Negative values on the off diagonals signify disagreement and vary in intensity of blue, whereas positive values on the diagonal represent agreement and vary in intensity of orange. For example, the cell at the intersection of BMI Z-score "Obese" and MUAC-Cl Staff "At Risk, Over" is darker blue because there is 50\% disagreement (1/2 measurements misclassified at that level). In contrast, BMI Z-score "Normal" and MUAC tape "At Risk, Over" is very light blue because the disagreement at that level is only 1\% (4/461 misclassified at that level) 


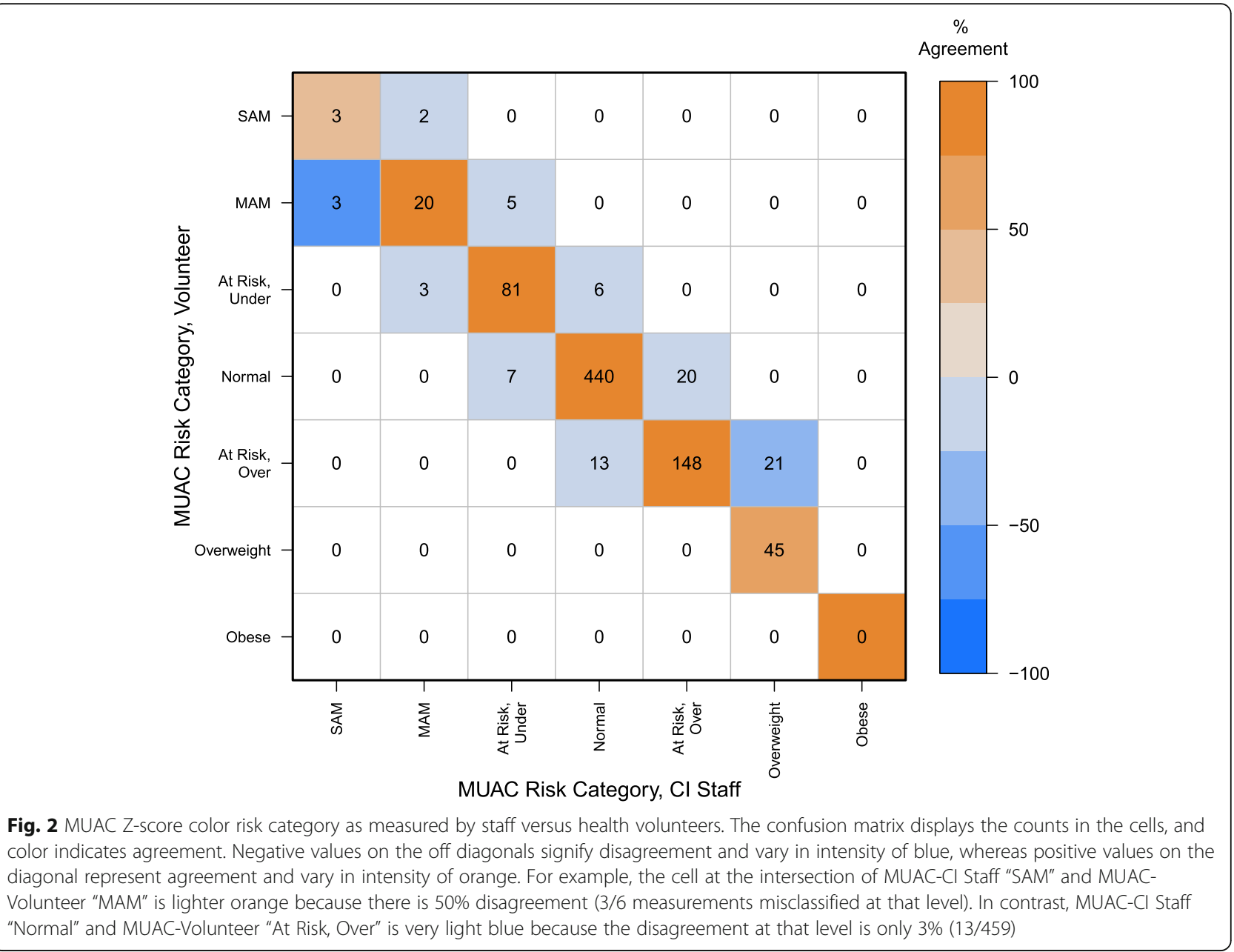

identify, all of which were labeled as "at risk" of undernutrition according to BMI z-score.

Model comparison tests determined that the model with a cubic term for BMI Z-score did not significantly improve model fit $\left(X^{2}(1)=0.85, P=0.36\right)$, but the quadratic model was significantly better than the linear model $\left(X^{2}(1)=43.70, P<0.0001\right)$. The results of the generalized linear mixed effect model revealed no significant association between age in months and odds of agreement between BMI Z-score and MUAC Z-score categories (OR: $1.05 ; 95 \%$ CI $(0.84,1.33) ; P=0.65)$. Gender was also not a significant factor affecting the odds of agreement (OR: 0.92; 95\% CI $(0.58,1.44) ; P=0.70)$. While there was no evidence that height-for-age Z-score was associated with odds of agreement (OR: 0.87, 95\% CI $(0.68,1.09)$, $P=0.23$ ), BMI Z-score was significantly associated with agreement in a quadratic curvilinear manner (Fig. $4, P<$ 0.0001). On average, a one-unit increase in BMI Z-score (i.e., 1 standard deviation) resulted in $79.44 \%$ increase in the $y$-x slope in odds of agreement (OR: 1.79, 95\% CI $(1.52,2.14))$. Effectively, this means that probability of disagreement increases at the extremes of BMI Z-score.

\section{Tierra Nueva qualitative results}

Surveys were completed by all 56 volunteers who contributed measurements to the study. Results of the survey questions appear in Table 3 . When asked how difficult it was to measure the circumference of the arm with the MUAC Z-score tape, 62.50\% (35/ 56) reported that it was either "easy" or "very easy." A similar proportion (34/56) reported that it was either "easy" or "very easy" to understand nutritional risk status with the tape. Seventy-one percent of respondents (40/56) preferred the MUAC Z-score tape to the traditional method of height and weight with an additional $25.00 \%$ reporting that they did not have sufficient information with respect to other methods to make a decision (14/56). Respondents had the opportunity to provide other comments or suggestions for improvement. The most common comment about how to improve the tape was the suggestion to change in the material (24/56, 42.86\%). Respondents also mentioned that the device should be available in the users' native language, in our case Spanish (3/56, 5.36\%). 


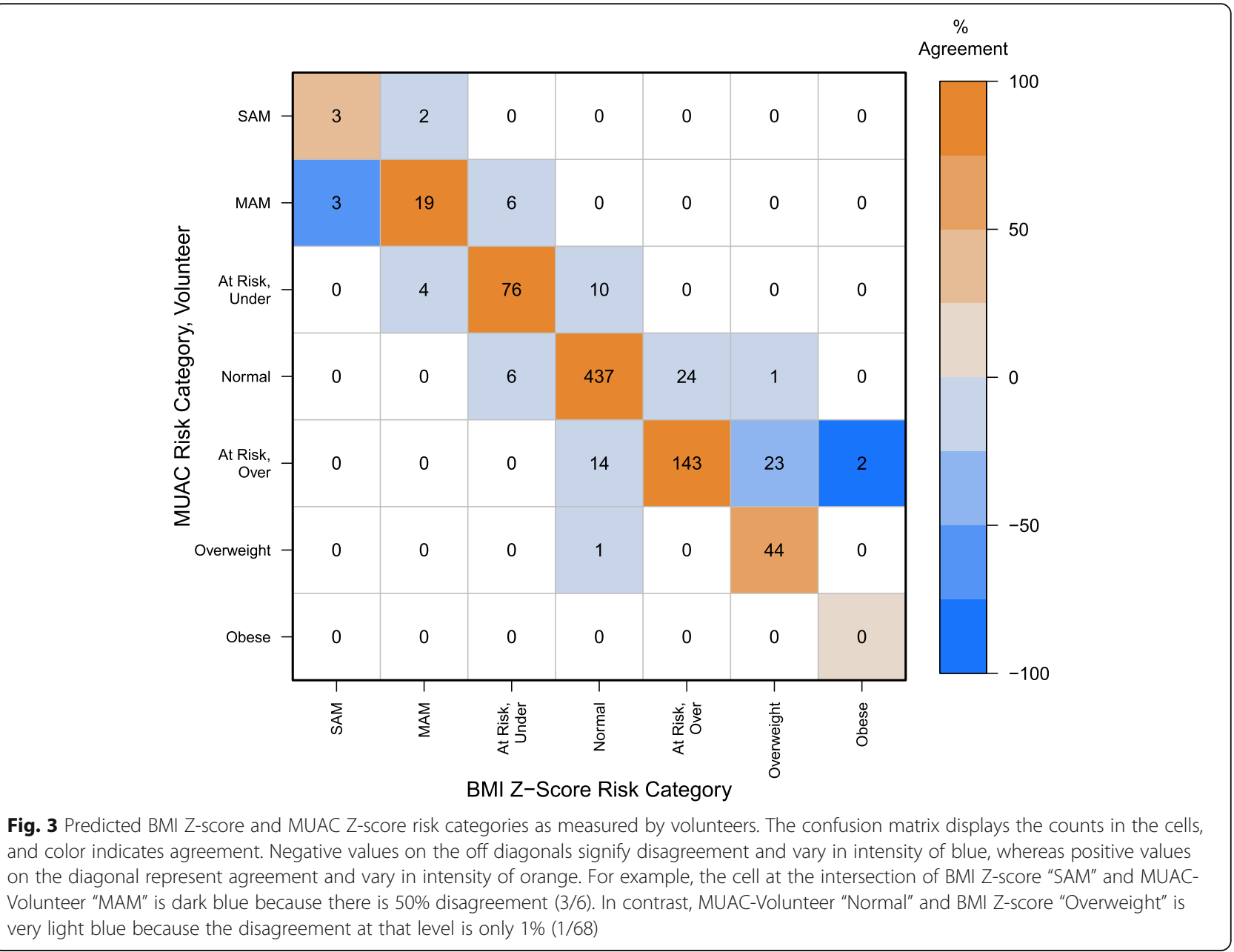

\section{Discussion}

The quantitative and qualitative findings suggest that the paper-based MUAC Z-score tape is a viable, low-cost alternative for assessing nutritional status among the community served by Children International in Guatemala. The present method of identifying cases of moderate and severe acute malnutrition (BMI Z-score) places the onus on time-constrained medical personnel and other field staff with little or no medical experience. It also requires special equipment that must either be taken into rural communities or kept at a community center that is often

Table 2 Confusion matrix for nutritional rehabilitation program candidates

\begin{tabular}{|c|c|c|c|}
\hline & \multicolumn{3}{|c|}{ MUAC Tape Color-Volunteer } \\
\hline & & Not a candidate & Candidate \\
\hline \multirow[t]{2}{*}{ BMI Z-score } & Not a candidate & 781 & 6 \\
\hline & Candidate & 4 & 27 \\
\hline
\end{tabular}

Candidates would fall in the two lowest categories ( -2 SD on either scale, BMI Z-score or MUAC Z-score) many miles from the homes of beneficiaries. The singlestep MUAC Z-score tape is much faster and easier to understand than collecting height and weight, calculating $\mathrm{BMI}$, converting BMI to an age- and gender-relevant Zscore, and assigning nutritional risk based on that value.

These results further suggest that MUAC Z-score measurements completed by a corps of health volunteers is an effective way to screen for malnutrition. Volunteers with little to no medical experience were able to achieve $91.00 \%$ agreement with field medical staff, $88.26 \%$ agreement with BMI Z-scores, and $87.10 \%$ agreement in identifying underweight candidates for the nutrition rehabilitation program according to BMI Z-scores. However, only about half of the individuals with SAM were identified by both methods (Fig. 3, top left corner). While there are few observations in the extreme nutritional risk categories on either end, the model results provide evidence that probability of disagreement is more likely to occur the more extreme the measurement. This finding is consistent with a previous observation in U.S. children which concluded that Z-score thresholds may require refinement to improve concordance [33]. 
Table 3 Survey results from the volunteers who contributed to the study

\begin{tabular}{|c|c|c|c|c|c|c|c|c|c|}
\hline \multicolumn{2}{|c|}{$\begin{array}{l}\text { How easy was it to measure } \\
\text { MUAC using this tape? }\end{array}$} & \multicolumn{2}{|c|}{$\begin{array}{l}\text { How easy was it to understand the } \\
\text { nutrition risk using this tape? }\end{array}$} & \multicolumn{2}{|c|}{$\begin{array}{c}\text { How helpful was this tape in making } \\
\text { decisions for intervention? }\end{array}$} & \multicolumn{2}{|c|}{$\begin{array}{l}\text { How does this tape compare to other } \\
\text { methods for assessing nutrition }\end{array}$} & \multicolumn{2}{|c|}{$\begin{array}{l}\text { How many times have you used } \\
\text { this tape? }\end{array}$} \\
\hline Very easy & $16.1 \%$ & Very easy & $8.9 \%$ & Very helpful & $69.6 \%$ & $\begin{array}{l}\text { I prefer this tape to } \\
\text { other methods }\end{array}$ & $71.4 \%$ & $0-10$ & $66.1 \%$ \\
\hline $\begin{array}{l}\text { Somewhat } \\
\text { easy }\end{array}$ & $46.4 \%$ & $\begin{array}{l}\text { Somewhat } \\
\text { easy }\end{array}$ & $51.8 \%$ & $\begin{array}{l}\text { Somewhat } \\
\text { helpful }\end{array}$ & $25.0 \%$ & & & $10-25$ & $32.1 \%$ \\
\hline $\begin{array}{l}\text { Neither easy } \\
\text { nor difficult }\end{array}$ & $37.5 \%$ & $\begin{array}{c}\text { Neither easy } \\
\text { nor difficult }\end{array}$ & $26.8 \%$ & I'm not sure & $5.4 \%$ & $\begin{array}{c}\text { I don't have enough } \\
\text { information to make this } \\
\text { decision }\end{array}$ & $25.0 \%$ & $25-50$ & $1.8 \%$ \\
\hline $\begin{array}{l}\text { Somewhat } \\
\text { difficult }\end{array}$ & $0.0 \%$ & $\begin{array}{l}\text { Somewhat } \\
\text { difficult }\end{array}$ & $12.5 \%$ & $\begin{array}{l}\text { Somewhat } \\
\text { unhelpful }\end{array}$ & $0.0 \%$ & & & $>30-40$ & $0.0 \%$ \\
\hline Very difficult & $0.0 \%$ & Very difficult & $0.0 \%$ & $\begin{array}{c}\text { Very } \\
\text { unhelpful }\end{array}$ & $0.0 \%$ & $\begin{array}{l}\text { I prefer the traditional } \\
\text { method using a scale } \\
\text { and height ruler }\end{array}$ & $3.6 \%$ & & \\
\hline
\end{tabular}

Importantly, this prior investigation also reported rates of concordance between both BMI Z-score and MUAC Zscore with clinical assessments of malnutrition raising questions of which measure is more sensitive for malnutrition [33]. There was no significant association between gender, age, or stunting (height-for-age Z-score $<-2$ ) with probability of disagreement. Only BMI Z-score (quadratic term) was found to be associated with probability of disagreement.

Our findings contribute to the current body of knowledge concerning the effectiveness of MUAC Z-score tape in identifying malnutrition in school-aged children and adolescents in the following ways. Firstly, this study represents one of the first instances of external validation of age-specific MUAC Z-scores using the novel tape in children aged 5 to 19 years in a community setting. Second, these results suggest that measurements completed by volunteers with little to no medical experience are in high agreement with those completed by the field medical staff and with the nutritional risk status as predicted BMI Z-scores. Third, a qualitative survey showed that the MUAC Z-score tape is easy to use and increases understanding of nutritional risk, although there is room for improvement in training and in the characteristics of the tape itself.

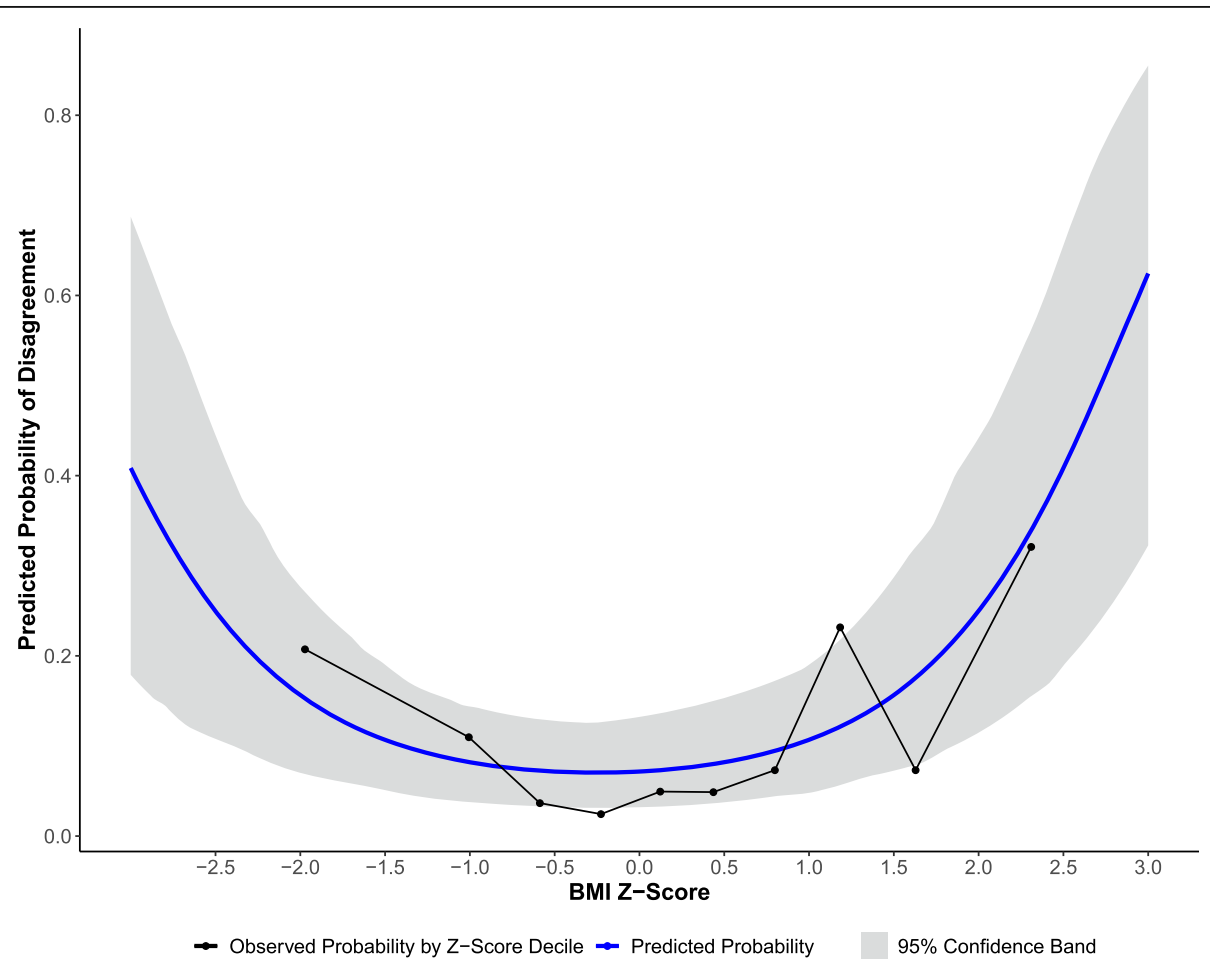

Fig. 4 Model predicted versus observed probability of disagreement. The figure shows the model results between BMI Z-score and MUAC Z-score measured by volunteers (by BMI Z-score quantiles) 
Field staff in Guatemala noted that the MUAC Z-score measurements which were discordant with BMI Z-scores occurred at the borders of two colors. This misclassification of some of the MAM and SAM cases indicates that a protocol to classify borderline cases may be required to enhance concordance between the measures. Other possible solutions include repeating measurements, or measuring the opposite arm as well and recording the more extreme of the two. Also noted, the nature of discordance did depend on nutritional status to some extent. MUAC Z-score was more likely to categorize an undernourished child into a more severe classification and an overnourished child into a less severe classification, a finding that coincides with previous studies [33]. Recommendations from the Guatemala field staff to improve the training and ultimately the effectiveness of the MUAC Z-score tape in a community setting include offering a reference pamphlet to the volunteers, which shows the exact version of the MUAC Z-score tape are using, with both sides and corresponding colors. They also noted that the practice sessions were very helpful and one of the most important aspects of the training. Having a doctor, nutritionist, or someone with medical experience leading the process was important during the training. Another benefit of the trainings noted by the field staff was that empowering volunteers and community leaders who have no previous formal medical training foments trust that may improve the quality and efficiency of community projects.

Of note in this study was the high prevalence of "at risk" of overweight and overweight. In contrast to other countries in Latin America where overweight and obesity are rapidly increasing, Guatemala has one of the lowest prevalences of overweight, and one of the lowest prevalences of inactive people over the age of 15 [39]. However, other studies have pointed to the nutrition transition that is occurring in Guatemala toward processed (e.g., canned foods, cheeses, refined sugar) and highly processed foods (e.g., chips, soft drinks, sweetened breakfast cereals) is associated with increases in BMI $[40,41]$. Because Guatemala has such a high rate of childhood stunting, the risk of the double burden of malnutrition (overweight or obesity coexisting with undernutrition) is alarming [42]. The MUAC Z-score tape used in this study not only identifies underweight, but the entire spectrum of nutritional status. The use of this specific tape has indicated that there may be a growing concern of overweight and obesity in the population of children and youth served in Guatemala, which may have implications for the field medical staff and nutrition program.

There are several limitations of the current study. One limitation is that the community volunteers were asked to measure children and youth in their communities, and were not randomly assigned participants. This may have introduced selection bias, especially if children were related or lived in close proximity, or if those children or youth who were not included were fundamentally different in ways that were related to the outcome of agreement. Additionally, there were 71 children or youth who did not complete the follow-up measurements, which could have skewed the results. Another limitation is the window of time between MUAC Z-score tape measurement and height and weight collection. Weight is affected by diet, activity level, and illness. The extent to which these environmental factors may have changed a child's weight and thus BMI over the course of 15 days were not recorded in this study; however, their impact is likely low in this narrow timeframe. When considering the limitations of the generalized linear mixed model, we had far fewer observations in the extreme tails of the distribution than near the center, making those estimates less certain. Interpretation near the ends of or beyond the observed range of BMI Z-scores should be done with caution. We also did not have a determination of nutritional status as determined by a medical professional; we compared only anthropometric measurements. Therefore, knowing the accuracy or performance of each method is not known, but as screening methods they yield similar results. Lastly, the MUAC Z-score tape collates MUAC Z-score data delineated by age in months into $1 / 2$ year or full year increments. Practical implications limit the real estate available on the MUAC Zscore tape, thus the age categories are not as granular; however, the tapes can be customized to expand or contract age ranges as was done for Children International.

\section{Conclusions}

To the best of our knowledge, this is the first study to assess the effectiveness of the MUAC Z-score tape in a community setting using non-medical volunteers. Volunteer-implemented nutritional risk screening with the MUAC Z-score tape was effective at detecting SAM and MAM in this study.

Future directions for this work include investigating scalability of the MUAC Z-score tape to detect malnutrition in locations such as Guatemala that require cost-effective strategies to address the heavy burden. Use of MUAC Z-score tape to monitor rehabilitation progress and determine success also remains to be investigated.

\section{Supplementary information}

Supplementary information accompanies this paper at (https://doi.org/10. 1186/s13690-019-0370-0).

Additional file 1: MUAC Z-Score Tape Instructions for Use. This reference document contains the instructions (English) and diagrams of portions of the MUAC Z-score tape to facilitate training and interpretation. (PDF 1726 kb) 


\section{Abbreviations}

BMl: Body Mass Index; Cl: Children International; MAM: Moderate Acute Malnutrition; MUAC: Mid-Upper Arm Circumference; SAM: Severe Acute Malnutrition; WH: Weight for Height; WHO: World Health Organization

\section{Acknowledgements}

Not applicable.

\section{Author's contributions}

MAM analyzed the data and produced the manuscript text. KM aided in the design of the experiment and data collection tools, and translated training materials into Spanish. ME and ACT were responsible for training the volunteers and organizing field staff to ultimately carry out the study in Guatemala. SAR facilitated development and procurement of the device for use in this study, and provided manuscript review and guidance.

\section{Funding}

This work was supported in part by a grant from the New England Pediatric Device Consortium.

\section{Availability of data and materials}

The datasets used and/or analyzed during the current study are available from the corresponding author on reasonable request. Information regarding the MUAC Z-score tape and instructions for use are also available upon request.

\section{Ethics approval and consent to participate}

This study was conducted under a protocol that was reviewed and approved by the Institutional Review Board of Children's Mercy Hospital (IRB\# 15040158).

\section{Consent for publication}

Not applicable to this research article.

\section{Competing interests}

MAM, KM, ME, and ACT have no competing interests. As the inventor of the MUAC Z-score device, SAR reports that a U.S. patent 10,238,317 has been issued to her employer.

\section{Author details}

${ }^{1}$ Children International, Kansas City, USA. ${ }^{2}$ Children International, Guatemala City, Guatemala. ${ }^{3}$ Children's Mercy Hospital, Kansas City, USA.

Received: 31 May 2019 Accepted: 3 September 2019

Published online: 04 October 2019

\section{References}

1. Black RE, Victora CG, Walker SP, Qar A, Bhutta Z, Christian P, De Onis M, et al. Maternal and child nutrition 1 maternal and child undernutrition and overweight in low-income and middle-income countries. Lancet. 2013:382:427-51. https://doi.org/10.1016/S0140-6736(13)60937-X.

2. Nyirenda H, Lelijveld N, Seal A, Wells JC, Kirkby J, Opondo C, et al. Chronic disease outcomes after severe acute malnutrition in Malawian children (ChroSAM): a cohort study. Artic Lancet Glob Heal. 2016;4:654-62. https:// doi.org/10.1016/S2214-109X(16)30133-4

3. Bwakura-Dangarembizi M, Amadi B, Bourke CD, Robertson RC, Mwapenya B, Chandwe $\mathrm{K}$, et al. Health outcomes, pathogenesis and epidemiology of severe acute malnutrition (HOPE-SAM): rationale and methods of a longitudinal observational study the HOPE-SAM study team. BMJ Open. 2019;9:23077. https://doi.org/10.1136/bmjopen-2018-023077.

4. Dewey KG, Begum K. Long-term consequences of stunting in early life. Matern Child Nutr. 2011;7:5-18. https://doi.org/10.1111/j.1740-8709.2011. 00349.x.

5. Prentice AM, Ward KA, Goldberg GR, Jarjou LM, Moore SE, Fulford AJ, et al. Critical windows for nutritional interventions against stunting 1-3. Am J Clin Nutr. 2013:97:911-8. https://doi.org/10.3945/ajcn.112.052332.

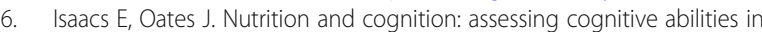
children and young people. Eur J Nutr. 2008:47:4-24. https://doi.org/10. 1007/s00394-008-3002-y.
7. Kim JY, Kang SW. Relationships between Dietary Intake and Cognitive Function in Healthy Korean Children and Adolescents. J Lifestyle Med. 2017;7:10-7. https://doi.org/10.15280/jlm.2017.7.1.10

8. Best C, Neufingerl N, van Geel L, van den Briel T, Osendarp S. The nutritional status of school-aged children: why should we care? Food Nutr Bull. 2010;31:400-17.

9. World Bank. Repositioning nutrition as central to development a strategy for large-scale action. 2006

10. Martins VJB, Toledo Florê Ncio TMM, Grillo LP, Do M, Franco CP, Martins PA, et al. Long-lasting effects of undernutrition. Int J Environ Res Public Heal. 2011;8:1817-46. https://doi.org/10.3390/ijerph8061817.

11. Jackson A, Ashworth A. The development of capacity at scale. Food Nutr Bull. 2015:36(Supplement):S47-52.

12. Van Tonder E, Mace L, Steenkamp L, Tydeman-Edwards R, Gerber K, Friskin D. Mid-upper arm circumference (MUAC) as a feasible tool in detecting adult malnutrition. South African J Clin Nutr. 2018:1-6. https://doi.org/10. 1080/16070658.2018.1484622

13. Jackson AA. Identifying children at risk of malnutrition. Nutr J. 2018;17:1-4 https://doi.org/10.1186/s12937-018-0392-4.

14. Grellety E, Golden MH. Severely malnourished children with a low weightfor-height have a higher mortality than those with a low mid-upper-armcircumference: I. empirical data demonstrates Simpson's paradox. Nutr J. 2018;17:1-21. https://doi.org/10.1186/s12937-018-0384-4

15. WHO | BMI-for-age (5-19 years). WHO 2019. https://www.who.int/growthref/ who2007_bmi_for_age/en/ ().

16. Roberfroid D, Hammami N, Lachat C, Prinzo ZW, Sibson V, Guesdon B, et al. Utilization of mid-upper arm circumference versus weight-for-height in nutritional rehabilitation programmes: a systematic review of evidence. 2013.

17. Chiabi A, Mbanga C, Mah E, Dongmo FN, Nguefack S, Fru F, et al. Weightfor-height Z score and mid-upper arm circumference as predictors of mortality in children with severe acute malnutrition. J Trop Pediatr. 2017;63: 260-6. https://doi.org/10.1093/tropej/fmw083.

18. Grellety E, Krause LK, Shams Eldin M, Porten K, Isanaka S. Comparison of weight-for-height and mid-upper arm circumference (MUAC) in a therapeutic feeding programme in South Sudan: is MUAC alone a sufficient criterion for admission of children at high risk of mortality? Public Health Nutr. 2015;18:2575-81. https://doi.org/10.1017/S1368980015000737.

19. Briend A, Maire B, Fontaine O, Garenne M. Mid-upper arm circumference and weight-for-height to identify high-risk malnourished under-five children. Matern Child Nutr. 2012;8:130-3. https://doi.org/10.1111/j.1740-8709.2011.00340.x.

20. Hossain I, Ahmed T, El Arifeen S, Billah SM, Faruque A, Islam MM, et al. Comparison of midupper arm circumference and weight-for-height z score for assessing acute malnutrition in Bangladeshi children aged 6-60 mo: an analytical study. Am J Clin Nutr. 2017;106:1232-9. https:/doi.org/10.3945/ajcn.

21. Goossens S, Bekele Y, Yun O, Harczi G, Ouannes M, Shepherd S. Mid-upper arm circumference based nutrition programming: evidence for a new approach in regions with high burden of acute malnutrition. PLOS One. 2012;7:e49320. https://doi.org/10.1371/journal.pone.0049320.

22. Fiorentino M, Sophonneary $P$, Laillou A, Whitney $S$, de Groot R, Perignon M, et al. Current MUAC cut-offs to screen for acute malnutrition need to be adapted to gender and age: the example of Cambodia. PLoS One. 2016;11:e0146442. https://doi.org/10.1371/journal.pone.0146442.

23. Sultana T, Nazmul Karim M, Ahmed T, lqbal HM. Assessment of under nutrition of Bangladeshi adults using anthropometry: can body mass index be replaced by mid-upper-arm-circumference? PLoS One. 2015;10:-e0121456. https://doi.org/10.1371/journal.pone.0121456

24. Sachdeva S, Dewan P, Shah D, Malhotra RK, Gupta P. Mid-upper arm circumference $\mathrm{v}$. weight-for-height Z-score for predicting mortality in hospitalized children under 5 years of age. Public Health Nutr. 2016;19: 2513-20. https://doi.org/10.1017/S1368980016000719.

25. Mogendi JB, De Steur H, Gellynck X, Saeed HA, Makokha A. Efficacy of midupper arm circumference in identification, follow-up and discharge of malnourished children during nutrition rehabilitation. Nutr Res Pract. 2015;9: 268. https://doi.org/10.4162/nrp.2015.9.3.268

26. Fernandez MAL, Delchevalerie $P$, van Herp M. Accuracy of MUAC in the detection of severe wasting with the new WHO growth standards. Pediatrics. 2010;126:e195-201. https://doi.org/10.1542/peds.2009-2175.

27. Mantilla-Hernández LC, Niño-Bautista L, Prieto-Pinilla EE Validating midupper arm circumference (MUAC) measurement for detecting acute malnutrition in 6-59 month-old children in emergency and disaster situations. Rev Salud Pública. 2014;16:195-207. 
28. Cordovés P, Cuan H, Viamontes $\mathrm{O}$, Pérez M, Pérez M. Perímetro braquial para diagnosticar estado nutricional en niños de dos a cinco años. Rev Argentina Anatomía Online. 2014;5:107-13.

29. Fierro PM, Sanchez IY, Chinchilla M. Tobar LF. Frente a Los Indicadores Talla Para La Edad, Peso Para La Edad, y Peso Para La Talla En Las Comunidades Indigenas, Poblaciones Afrocolombianas y Mestizas: El Perímetro Braquial Para Diagnosticar Estado Nutricional; n.d. https://encolombia.com/medicina/ materialdeconsulta/tensiometro-virtual/perimetro-branquial/ (

30. Mramba L, Ngari M, Mwangome M, Muchai L, Bauni E, Walker AS, et al. A growth reference for mid upper arm circumference for age among school age children and adolescents, and validation for mortality: growth curve construction and longitudinal cohort study. BMJ. 2017;358:33423. https://doi. org/10.1136/BMJ.J3423.

31. Addo OY, Himes JH, Zemel BS. Reference ranges for midupper arm circumference, upper arm muscle area, and upper arm fat area in US children and adolescents aged 1-20 y. Am J Clin Nutr. 2017;105:111-20. https://doi.org/10.3945/ajcn.116.142190.

32. Abdel-Rahman SM, Bi C, Thaete K. Construction of lambda, mu, sigma values for determining mid-upper arm circumference $z$ scores in U.S. children aged 2 months through 18 years. Nutr Clin Pract. 2017;32:68-76. https://doi.org/10.1177/0884533616676597.

33. Stephens K, Escobar A, Jennison EN, Vaughn L, Sullivan R, Abdel-Rahman S, et al. Evaluating mid-upper arm circumference Z-score as a determinant of nutrition status. Nutr Clin Pract. 2018;33:124-32. https://doi.org/10.1002/ncp. 10018.

34. Bliss J, Lelijveld N, Briend A, Kerac M, Manary M, McGrath M, et al. Use of mid-upper arm circumference by novel community platforms to detect, diagnose, and treat severe acute malnutrition in children: a systematic review. Glob Heal Sci Pract. 2018;6:552-64. https://doi.org/10.9745/GHSP-D18-00105.

35. Alé $F G B$, Phelan $K P Q$, Issa H, Defourny I, Le Duc G, Harczi G, et al. Mothers screening for malnutrition by mid-upper arm circumference is non-inferior to community health workers: results from a large-scale pragmatic trial in rural Niger. Arch Public Health. 2016;74:38. https://doi.org/10.1186/s13690016-0149-5.

36. WHO | WHO Anthro (version 3.2.2, January 2011) and macros. WHO 2017.

37. Efron B. Better bootstrap confidence intervals. J Am Stat Assoc. 1987;82:171. https://doi.org/10.2307/2289144.

38. Martinez B, Webb MF, Gonzalez A, Douglas K, Grazioso MDP, Rohloff P. Complementary feeding intervention on stunted Guatemalan children: a randomised controlled trial. BMJ Paediatr Open. 2018;2:213. https://doi.org/ 10.1136/bmjpo-2017-000213.

39. Corvalán C, Garmendia ML, Jones-Smith J, Lutter CK, Miranda JJ, Pedraza LS, et al. Nutrition status of children in Latin America. Obes Rev. 2017;18:7-18. https://doi.org/10.1111/obr.12571

40. Asfaw A. Does consumption of processed foods explain disparities in the body weight of individuals? The case of Guatemala. Health Econ. 2011:20:184-95. https://doi.org/10.1002/hec.1579.

41. Moubarac JC, Batal M, Martins APB, Claro R, Levy RB, Cannon G, et al. Processed and ultra-processed food products: consumption trends in Canada from 1938 to 2011. Can J Diet Pract Res. 2014;75:15-21. https://doi. org/10.3148/75.1.2014.15.

42. Ramirez-Zea M, Kroker-Lobos MF, Close-Fernandez R, Kanter R. The double burden of malnutrition in indigenous and nonindigenous Guatemalan populations. Am J Clin Nutr. 2014;100:1644S-51S. https://doi.org/10.3945/ ajcn.114.083857.

\section{Publisher's Note}

Springer Nature remains neutral with regard to jurisdictional claims in published maps and institutional affiliations.

Ready to submit your research? Choose BMC and benefit from:

- fast, convenient online submission

- thorough peer review by experienced researchers in your field

- rapid publication on acceptance

- support for research data, including large and complex data types

- gold Open Access which fosters wider collaboration and increased citations

- maximum visibility for your research: over $100 \mathrm{M}$ website views per year

At BMC, research is always in progress.

Learn more biomedcentral.com/submissions 